\title{
Comparison of Physician-recorded Toxicities and Patient-reported Outcomes of Five Different Radiotherapy Methods for Prostate Cancer
}

\author{
MAKOTO ITO ${ }^{1,2}$, KAZUMA SASAMURA $^{3}$, YUUKI TAKASE ${ }^{2,4}$, TADAYUKI KOTSUMA ${ }^{5,6}$, \\ YUKIHIKO OSHIMA $^{1}$, YOSHITAKA MINAMI ${ }^{1}$, JUNJI SUZUKI ${ }^{2}$, EIICHI TANAKA ${ }^{5}$, WATARU OHASHI ${ }^{7}$, \\ MASAHIKO OGUCHI ${ }^{3}$, TAKAHITO OKUDA ${ }^{2}$, KOJIRO SUZUKI $^{1}$ and YASUO YOSHIOKA ${ }^{2,3}$ \\ ${ }^{1}$ Department of Radiology, Aichi Medical University Hospital, Nagakute, Japan; \\ ${ }^{2}$ Department of Radiation Oncology, Toyota Memorial Hospital, Toyota, Japan; \\ ${ }^{3}$ Radiation Oncology Department, Cancer Institute Hospital of Japanese Foundation for Cancer Research, Tokyo, Japan; \\ ${ }^{4}$ Department of Radiology, Nagoya University Hospital, Nagoya, Japan; \\ ${ }^{5}$ Department of Radiation Oncology, National Hospital Organization Osaka National Hospital, Osaka, Japan; \\ ${ }^{6}$ Department of Radiology, Kaizuka City Hospital, Kaizuka, Japan; \\ ${ }^{7}$ Department of Biostatistics, Clinical Research Center, Aichi Medical University Hospital, Nagakute, Japan
}

\begin{abstract}
Background/Aim: To compare five radiotherapy methods for prostate cancer. Patients and Methods: During 2005-2018, the data of patients with non-metastatic prostate cancer were retrospectively analysed. Patients were treated with high-dose-rate brachytherapy (HDR-BT); low-dose-rate brachytherapy (LDR-BT); or external-beam radiotherapy $(E B R T)$, including conventionally fractionated radiotherapy (CFRT), moderate-hypofractionated radiotherapy (MHRT), and ultra-hypofractionated radiotherapy (UHRT). Results: In total, 496 patients (149, HDR-BT; 100, LDR-BT; 100, CFRT; 97, MHRT, and 50, UHRT) with a median follow-up of 4.3 years were enrolled. The incidence of grade $\geq 2$ acute genitourinary toxicities was significantly lower with HDR$B T(p<0.001)$ than with any other radiotherapy. The cumulative incidence of late grade $\geq 2$ genitourinary toxicities was the highest with UHRT and significantly higher $(p=0.005)$ with UHRT than with HDR-BT. Higher symptom score peaks were noted 4 weeks after therapy for LDR-BT than for EBRT. Conclusion: Physician-recorded
\end{abstract}

This article is freely accessible online.

Correspondence to: Makoto Ito (ORCID: 0000-0001-7733-1594), MD, PhD, Department of Radiology, Aichi Medical University Hospital, 1-1 Yazako-Karimata, Nagakute, Aichi 480-1195, Japan. Tel: +81 561623311, Fax: +81 561633268, e-mail: itou.makoto.292@mail.aichi-med-u.ac.jp

Key Words: Radiotherapy, prostate cancer, brachytherapy, highdose-rate, low-dose-rate, hypofractionated radiotherapy. toxicities were slightly lower with HDR-BT and patientreported outcomes tended to be worse with LDR-BT.

Prostate cancer is the most prevalent malignancy in men across all countries (1). In addition to prostatectomy, radiotherapy is the standard therapeutic option. Brachytherapy (BT) and external beam radiotherapy (EBRT) are the main types of radiotherapy. BT has two main forms: high-dose-rate BT (HDR-BT) and low-dose-rate BT (LDR-BT).

For EBRT, conventionally fractionated radiotherapy (CFRT) administered as 1.8-2.0 Gy fractions (fx) at a typical total dose of 74-78 Gy has been the standard regimen for many years. However, in recent years, moderate-hypofractionated radiotherapy (MHRT) and ultra-hypofractionated radiotherapy (UHRT) with higher doses per fraction than that in conventional radiotherapy have also been used (2). Radiation oncologists need to be familiar with all these treatment approaches to provide optimal treatment for each patient.

Although risk stratification is a general treatment strategy, the guidelines have not recommended one radiotherapy technique over another (3). In most cases, BT and EBRT have shown comparable treatment efficacy (4-6). The differences in toxicities are therefore critical in treatment selection. Several retrospective studies have indicated that the occurrence of acute genitourinary (GU) toxicity was lower with HDR-BT than with LDR-BT, while late toxicity was similar $(5,7)$. In contrast, the occurrence of late gastrointestinal (GI) toxicity was lower in LDR-BT than in EBRT while that of late GU toxicity was equivalent or lower in EBRT than in $\operatorname{LDR}-\mathrm{BT}(8,9)$. The CHHiP trial revealed that, compared with EBRT, MHRT was non-inferior to CFRT regarding toxicity and efficacy (10). 
While the difference with respect to clinical results (toxicity and efficacy) between UHRT and CFRT continues to gain clarity with more published research $(2,11)$, very few studies have simultaneously compared these radiotherapy methods $(12,13)$. In particular, no direct comparison studies, with adequate data, between HDR-BT as monotherapy and hypofractionated EBRT have been published. Because direct comparisons can minimise observer bias, these types of data will be more robust than indirect comparisons of multiple studies. Therefore, in this study, we retrospectively reviewed the data of prostate cancer patients treated with the following five different radiotherapy methods: HDR-BT, LDR-BT, CFRT, MHRT, and UHRT. We also compared physicianrecorded toxicities and patient-reported outcomes after treatment with one of the five different radiotherapy methods, to enable the choice of the optimal radiotherapy method.

\section{Patients and Methods}

Patients. Medical records of non-metastatic prostate cancer (cT1-T4, N0, M0) patients from four institutions were reviewed retrospectively between 2005 and 2018. In this study, patients aged $\geq 20$ years who were treated with either BT or EBRT were included. The exclusion criteria were as follows: patients treated with palliative intent, those who received EBRT using three-dimensional conformal radiation therapy, those who received elective pelvic radiotherapy, those who received a combination of $\mathrm{BT}$ and EBRT, and those with a short follow-up ( $<1$ year). Finally, a total of 496 patients were included. Patients were classified into risk groups according to the National Comprehensive Cancer Network guidelines (3). Radiotherapy alone was offered to low-risk patients (clinical stage T1-T2a, prostatespecific antigen $[\mathrm{PSA}]<10 \mathrm{ng} / \mathrm{ml}$, and Gleason score of 6 ). In contrast, intermediate- to high-risk patients received neoadjuvant/adjuvant androgen deprivation therapy (ADT). ADT included luteinising hormone-releasing hormone agonists and/or anti-androgen, and it was used for longer periods in high-risk patients. This study was approved by the appropriate institutional review board, and the need for informed consent was waived.

Radiotherapy. Patients were treated with one of the following five radiotherapy methods in the four institutions: HDR-BT, LDR-BT, CFRT, MHRT, or UHRT. Patients in the EBRT group were treated with a linear accelerator or CyberKnife (Accuray Inc., Sunnyvale, CA, USA). Details of the radiotherapy methods are as follows.

HDR-BT. HDR-BT was performed as monotherapy using an HDR Iridium-192 source with $370 \mathrm{GBq}(10 \mathrm{Ci}$ ) activity. A total of $49 \mathrm{~Gy}$ in $7 \mathrm{fx}$ was delivered over four days. Treatment was accomplished within one implant session under continuous epidural anesthesia, and irradiation was administered twice daily with an interval of at least six hours. Needle insertion was performed under transrectal ultrasound guidance; however, the treatment planning was based on computed tomography (CT). The clinical target volume (CTV) included the whole prostate gland and medial seminal vesicles with a 0 - to 5-mm margin except for a 0 - to 3-mm margin for the rectal side.

In most cases, the planning target volume (PTV) equalled CTV, and the prescribed dose was delivered to $95 \%$ of the PTV (D95). Dose constraints for organs at risk were as follows: the maximum dose to the entire urethra should be $<125 \%$ of the prescription dose, preferably $<110 \%$, and the maximum dose to the entire rectal mucosa should be $<100 \%$ PD dose, preferably $<80 \%$. The dosevolume constraint for the rectum was D $5 \mathrm{cc}<55 \%$ of the prescription dose.

$L D R-B T$. LDR-BT was performed as monotherapy using I-125 seeds with mostly $13.1 \mathrm{MBq}(0.35 \mathrm{mCi})$ activity. The prescribed dose was 145 Gy. All patients underwent transrectal ultrasound preplanning 3-4 weeks before implantation to determine the number of seeds. Then, the actual implant was performed according to intraoperative planning under general anaesthesia. The CTV was defined as the prostate itself, which was also equal to the PTV. VariSeed 7.1 (Varian Medical Systems, Palo Alto, CA, USA) was used as the treatment planning system. The percent volumes of the PTV receiving $100 \%$ of the prescribed dose (V100\%) were planned to be $\geq 95 \%$, preferably $\geq 99 \%$. Dose constraints for organs at risk were as follows: V100\% for the rectum should be $=0 \%$, V $150 \%$ for the urethra should be $=0 \%$, and D30\% for the urethra should be $<200$ Gy, preferably $<180$ Gy. Post-planning using CT and magnetic resonance imaging was performed 1 month after treatment, and dosimetric parameters were evaluated. Note that patients in the LDR-BT group received only prophylactic alpha-blocker treatment for GU toxicity for approximately one year after radiotherapy.

CFRT. In principle, the prescribed dose was $78 \mathrm{~Gy}$ in $39 \mathrm{fx}$. In contrast, the dose for low-risk patients and/or those receiving anticoagulants was reduced to $74 \mathrm{~Gy}$ in $37 \mathrm{fx}$. The CTV was defined as the whole-prostate gland and proximal seminal vesicle, while the CTV was only the prostate in low-risk patients. The PTV1 included CTV with a 7-mm margin except for the rectal side, where a 4-mm margin was added. PTV2 was defined as the seminal vesicle with a 5-mm margin, excluding PTV1. The prescribed doses were delivered to PTV1 (D95), and 64 Gy were delivered to PTV2. Only in seminal vesicle invasion cases, 74 or 78 Gy were administered to both PTV1 and PTV2. The dose constraints were as follows: D2\% of the PTV should be $<107 \%$. V70 covers $<10 \%$ of the rectum, and V60 covers $<18 \%$. V70 covers $<16 \%$ of the entire bladder, and V60 covers $<25 \%$. D2\% of the penile bulb should be $<70 \%$. Both the target volume and normal organ structures were contoured using a treatment planning system (Eclipse, Varian Medical System). All patients were treated with a linear accelerator (TrueBeam STx, Varian Medical Systems).

MHRT. The prescription dose for MHRT was $70 \mathrm{~Gy}$ in $28 \mathrm{fx}$. The CTV was defined as the whole prostate gland and proximal seminal vesicle regardless of the risk group. Furthermore, the CTV included the entire seminal vesicle in seminal vesicle invasion cases. The PTV included the CTV with a 5-mm margin except for a 4-mm margin for the rectal side. The prescribed dose was adjusted to deliver $50 \%$ of the PTV (D50). The dose constraints were as follows: D95 of the PTV was $>95 \%$, and D2\% was $<105 \%$. The maximum dose to the rectum should be $<101 \%$, and the percentage covered by D20\% should be $<54 \%$ and that by V40\% should be $<37 \%$. The maximum dose to the entire bladder should be $<103 \%$, and the percentage covered by D20\% should be $<63 \%$ and that by $\mathrm{V} 40 \%$ should be $<28 \%$. Most patients $(\mathrm{n}=55)$ were treated with a linear accelerator, represented by TrueBeam (Varian Medical Systems). The Eclipse (Varian Medical System) was used for treatment planning. In contrast, 42 patients were treated using 
CyberKnife M6 (Accuray Inc.). All patients treated using CyberKnife were implanted with three gold fiducial markers owing to its incorporation of near real-time $\mathrm{kV}$ imaging of the prostate 2 weeks before treatment.

$U H R T$. A total dose of 36.25 Gy was prescribed for five consecutive days, excluding the days when the hospital was closed. The definition of CTV was the same as that in MHRT. Patients in the UHRT group had more thorough nutritional guidance and urinary/defecation management. Compared with other EBRT groups, the daily setup for UHRT was performed with greater care. Therefore, the CTV margin was very tight; the PTV included the CTV with a 5-mm margin except for a 3-mm margin for the rectal side. In addition, the part that overlapped with the rectal bladder mucosa was removed by $1 \mathrm{~mm}$. The prescription dose was adjusted to D95 with a $75 \%-85 \%$ isodose line to meet the dose constraints The dose constraints were as follows: the percentage of the rectum that should be covered by D $0.5 \mathrm{cc}$ was $<102 \%$ and that by D $5 \mathrm{cc}$ was $<77 \%$. The percentage of the entire bladder that should be covered by V50\% was $<35 \mathrm{cc}$ and that by V100\% was $<5 \mathrm{cc}$. Multiplan (Accuray Inc.) was used as the planning system. Treatment was performed using CyberKnife M6 (Accuray Inc.), except in three cases where TrueBeam STx was used. Only one periprostatic hydrogel spacer (SpaceOAR; Augmenix Inc., Boston, MA, USA) for EBRT was used in the UHRT group.

Evaluation of outcomes. The date of the event was measured from the date of commencement of radiotherapy. Biochemically, no evidence of disease (bNED) was defined according to the Phoenix definition, as an absolute nadir PSA level $+2 \mathrm{ng} / \mathrm{ml}$ increase (14).

Both physician-recorded toxicities and patient-reported outcomes were evaluated. Physician-recorded toxicities were assessed by four observers according to the National Cancer Institute Common Toxicity Criteria for Adverse Events (version 5.0). To reduce observer bias as much as possible, toxicity assessments were discussed in advance and only grade $\geq 2$ events were evaluated. Acute toxicity was defined as symptoms that were observed during or less than 6 months after radiotherapy treatment. Late toxicity was defined as any event persisting or occurring 6 months after completing radiotherapy. Patient-reported outcomes were evaluated to supplement the understanding of GU toxicity transitions, especially in the acute phase. These were evaluated based on the International Prostate Symptom Score (IPSS) and quality of life (QOL) score. The scores were recorded at the time of radiotherapy, then 1 week, 4 weeks, and 3 months, and thereafter every 3 months until 1 year after radiotherapy and were directly proportional to the patient's displeasure level.

Statistical analysis. All statistical analyses were performed using EZR version 1.33 (Saitama Medical Center, Jichi Medical University, Saitama, Japan) based on the R and R commander (The R Foundation for Statistical Computing, Vienna, Austria) (15). Multiple comparisons of percentages were performed using Fisher's exact tests with post-hoc Bonferroni analyses and a one-way analysis of variance (ANOVA) was performed to analyse the incidence acute toxicity. A comparison of IPSS transitions was performed using repeated-measures ANOVA. The Kaplan-Meier method was used to estimate bNED rates and the cumulative incidence of toxicities, and comparisons were performed using logrank tests or Gray's tests with post-hoc Bonferroni analyses. Cox's proportional hazard model was used for the univariate and multivariate analyses, which were used to determine the factors that contributed to the cumulative incidence of late GI/GU toxicity. $p$ Values $<0.05$ were considered statistically significant. Factors showing a difference with $p<0.1$ in the univariate analysis were included the multivariate analysis.

\section{Results}

Patient characteristics. The patient characteristics are listed in Table I. This study included 496 patients, with the HDR-BT, LDR-BT, CFRT, MHRT, and UHRT groups comprising 149, $100,100,97$, and 50 patients, respectively. There was no difference in age among the groups. The LDR-BT group included more low-risk patients than the other groups; therefore, the administration rate of ADT was lower, and the duration was shorter in the LDR-BT group than in the other groups. Patients in the EBRT and UHRT groups had the smallest planning target volume. More patients in the LDRBT and CFRT groups received medication for bladder symptoms than in the MHRT and UHRT groups. We were not able to confirm the medication rate in the HDR-BT group. The median follow-up period for all the 496 included patients was 4.3 years (range $=1.0-14.2$ years). The follow-up period was 10.0 years (range $=1.8-14.2$ years) in the LDR-BT group and 1.6 years (range $=1.0-2.5$ years) in the UHRT group.

Efficacy. The 3-year bNED rates of the total study population were $98.0 \%$ [ $95 \%$ confidence interval $(\mathrm{CI})=93.8-99.3 \%$ ], $99.0 \%(95 \% \mathrm{CI}=93.0-99.9 \%), 98.2 \%(95 \% \mathrm{CI}=88.0-99.7 \%)$, and $96.9 \%(95 \% \mathrm{CI}=87.7-99.3 \%)$ in the HDR-BT, LDR-BT, CFRT, and MHRT groups, respectively with no significant difference being observed $(p=0.403)$. While the follow-up period for patients in the UHRT group did not reach three years, biochemical failure was not observed. An analysis by risk group also showed no significant difference in bNED rates among patients in the five groups. A total of 466 patients (94\%) were alive at the time of analysis: 452 patients were alive with bNED, 13 were alive with biochemical failure only, and one was alive with distant metastasis. Only two patients died from prostate cancer, while 28 patients died because of other causes.

Acute toxicity. Grade 2 acute GI toxicity was found in three $(2 \%)$, two (2\%), two (2\%), eight $(9 \%)$, and $0(0 \%)$ patients in the HDR-BT, LDR-BT, CFRT, MHRT, and UHRT groups, respectively. The incidence of grade 2 acute GI toxicity in patients in the MHRT group was slightly higher, but no significant difference was observed for any of the other groups $(p>0.05)$. No grade $\geq 3$ acute GI toxicity was found in any of the patients from any of the groups. The incidence of grade $\geq 2$ acute GU toxicity was significantly lower in the HDR-BT group (13 cases, $8.7 \% ; p<0.001)$ than in the LDR-BT (31 cases, 31\%), CFRT (42 cases, 42\%), MHRT (31 cases, 32\%), 
Table I. Characteristics of patients with non-metastatic prostate cancer.

\begin{tabular}{|c|c|c|c|c|c|}
\hline Characteristic & HDR-BT & LDR-BT & CFRT & MHRT & UHRT \\
\hline Number of patients & 149 & 100 & 100 & 97 & 50 \\
\hline \multicolumn{6}{|l|}{ Age (years) } \\
\hline Median & 71 & 70 & 74 & 69 & 71 \\
\hline Range & $48-82$ & $53-84$ & $52-85$ & $53-84$ & $49-81$ \\
\hline \multicolumn{6}{|l|}{ iPSA (ng/ml) } \\
\hline Median & 9.8 & 7.2 & 8.7 & 9.2 & 8.0 \\
\hline Range & $2.0-149$ & $0.8-28.1$ & $4.1-190.2$ & $4.0-129.4$ & $4.2-54.2$ \\
\hline \multicolumn{6}{|l|}{ Gleason score } \\
\hline $4-6$ & $58(39 \%)$ & $69(69 \%)$ & $15(15 \%)$ & $24(25 \%)$ & $5(10 \%)$ \\
\hline 7 & $53(36 \%)$ & $31(31 \%)$ & $49(49 \%)$ & $62(64 \%)$ & $37(74 \%)$ \\
\hline $8-10$ & $38(25 \%)$ & $0(0 \%)$ & $36(36 \%)$ & $11(11 \%)$ & $8(16 \%)$ \\
\hline \multicolumn{6}{|l|}{$\mathrm{T}$ classification } \\
\hline 1 & $50(34 \%)$ & $61(61 \%)$ & $35(35 \%)$ & $37(38 \%)$ & $11(22 \%)$ \\
\hline 2 & $66(44 \%)$ & $39(39 \%)$ & $48(48 \%)$ & $36(37 \%)$ & $30(60 \%)$ \\
\hline $3 \mathrm{a}$ & $22(15 \%)$ & $0(0 \%)$ & $11(11 \%)$ & $22(23 \%)$ & $8(16 \%)$ \\
\hline $3 b$ & $9(6 \%)$ & $0(0 \%)$ & $4(4 \%)$ & $1(1 \%)$ & $1(2 \%)$ \\
\hline 4 & $2(1 \%)$ & $0(0 \%)$ & $2(2 \%)$ & $1(1 \%)$ & $0(0 \%)$ \\
\hline \multicolumn{6}{|l|}{ Risk group } \\
\hline Low & $27(18 \%)$ & $58(58 \%)$ & $12(12 \%)$ & $10(10 \%)$ & $4(8 \%)$ \\
\hline Intermediate & $58(39 \%)$ & $40(40 \%)$ & $46(46 \%)$ & $56(58 \%)$ & $35(70 \%)$ \\
\hline High & $64(43 \%)$ & $2(2 \%)$ & $42(42 \%)$ & $31(32 \%)$ & $11(22 \%)$ \\
\hline ADT & $121(81 \%)$ & $50(50 \%)$ & $87(87 \%)$ & $70(71 \%)$ & $46(92 \%)$ \\
\hline \multicolumn{6}{|l|}{ Duration before radiotherapy (months) } \\
\hline Median & 7.0 & 4.4 & 6.9 & 6.9 & 6.5 \\
\hline Range & $2.0-35.0$ & $1.5-15.0$ & $3.5-77.5$ & $1.5-93.6$ & $1.4-66.6$ \\
\hline \multicolumn{6}{|l|}{ Total duration (months) } \\
\hline Median & 8.0 & 4.4 & 17.6 & 9.3 & 6.8 \\
\hline Range & $2.0-48.0$ & $1.5-15.0$ & $3.5-77.5$ & $3.3-105.0$ & $1.4-66.6$ \\
\hline History of TURP & $0(0 \%)$ & $1(1 \%)$ & $1(1 \%)$ & $2(2 \%)$ & $2(4 \%)$ \\
\hline \multicolumn{6}{|l|}{ PTV volume $\left(\mathrm{cm}^{3}\right)$} \\
\hline Median & NA & NA & 68.1 & 56 & 45.6 \\
\hline Range & NA & NA & $47.5-230.9$ & $31.2-216.2$ & $26.4-90.4$ \\
\hline Medication for bladder symptoms at radiotherapy & NA & $21(21 \%)$ & $21(21 \%)$ & $12(12 \%)$ & $12(12 \%)$ \\
\hline Prescription dose $(\mathrm{Gy}) /$ fractions & $49 / 7$ & $145 /-$ & $74-78 / 37-39$ & $70 / 28$ & $36.25 / 5$ \\
\hline \multicolumn{6}{|l|}{ Median follow-up (years) } \\
\hline Median & 5.7 & 10.0 & 3.1 & 3.0 & 1.6 \\
\hline Range & $1.7-10.2$ & $1.8-14.2$ & $1.0-5.0$ & $1.0-9.5$ & $1.0-2.5$ \\
\hline
\end{tabular}

HDR-BT: High-dose rate brachytherapy; LDR-BT: low-dose rate brachytherapy; CFRT: conventional fractionated radiotherapy; MHRT: moderatehypofractionated radiotherapy; UHRT: ultra-hypofractionated radiotherapy; iPSA: initial prostate-specific antigen; ADT: androgen deprivation therapy; TURP: transurethral resection of the prostate; PTV: planning target volume; NA: not available.

Table II. Details of grade 2 acute genitourinary toxicities.

\begin{tabular}{|c|c|c|c|c|c|}
\hline & HDR-BT & LDR-BT & CFRT & MHRT & UHRT \\
\hline Total cases $(\%)$ & $13(8.7)$ & $31(31)$ & $42(42)$ & $31(32)$ & $20(40)$ \\
\hline Haematuria & 0 & 2 & 0 & 1 & 2 \\
\hline Urinary frequency & 0 & 25 & 21 & 15 & 6 \\
\hline Urinary incontinence & 1 & 2 & 4 & 3 & 0 \\
\hline Urinary retention & 5 & 25 & 7 & 11 & 12 \\
\hline Urinary urgency & 4 & 15 & 20 & 14 & 4 \\
\hline Urinary tract pain & 3 & 2 & 2 & 4 & 1 \\
\hline
\end{tabular}

HDR-BT: High-dose rate brachytherapy; LDR-BT: low-dose rate brachytherapy; CFRT: conventional fractionated radiotherapy; MHRT: moderatehypofractionated radiotherapy; UHRT: ultra-hypofractionated radiotherapy. 


\section{IPSS}

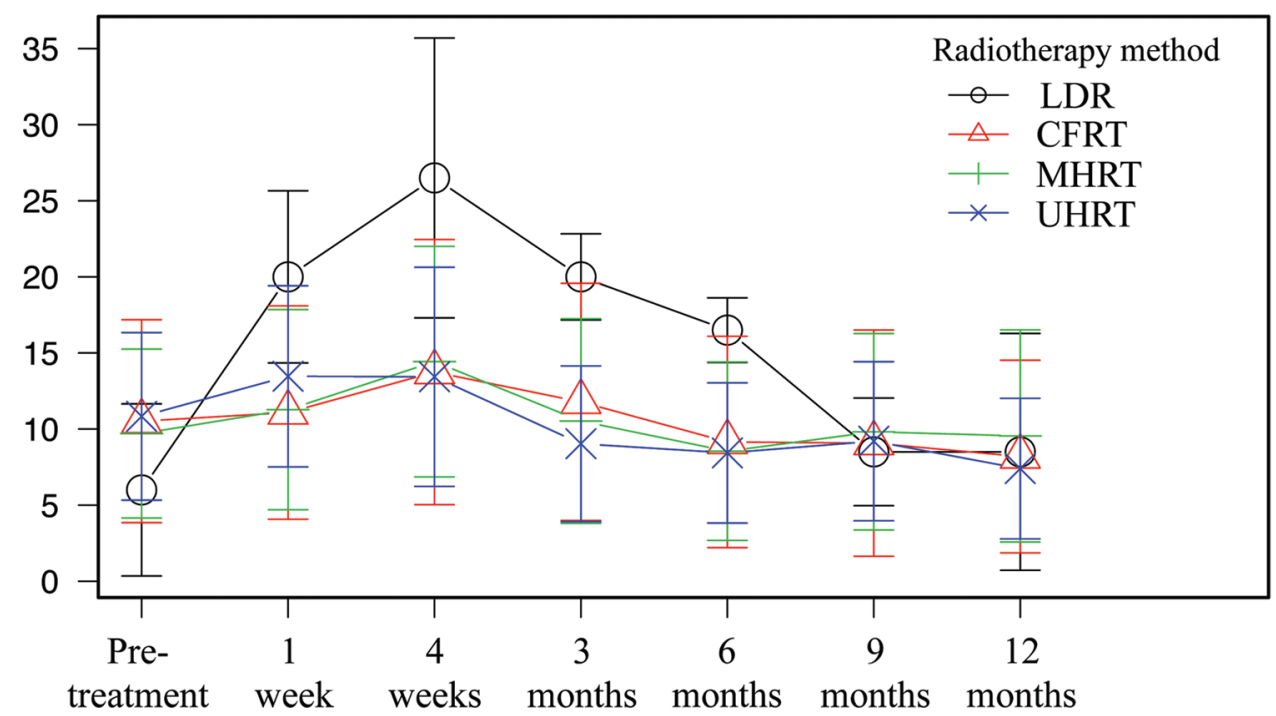

Figure 1. Changes in the IPSS according to radiotherapy methods. IPSS: International prostate symptom score; LDR: low-dose rate; CFRT: conventional fractionated radiotherapy; MHRT: moderate-hypofractionated radiotherapy; UHRT: ultra-hypofractionated radiotherapy.

\section{QOL score}

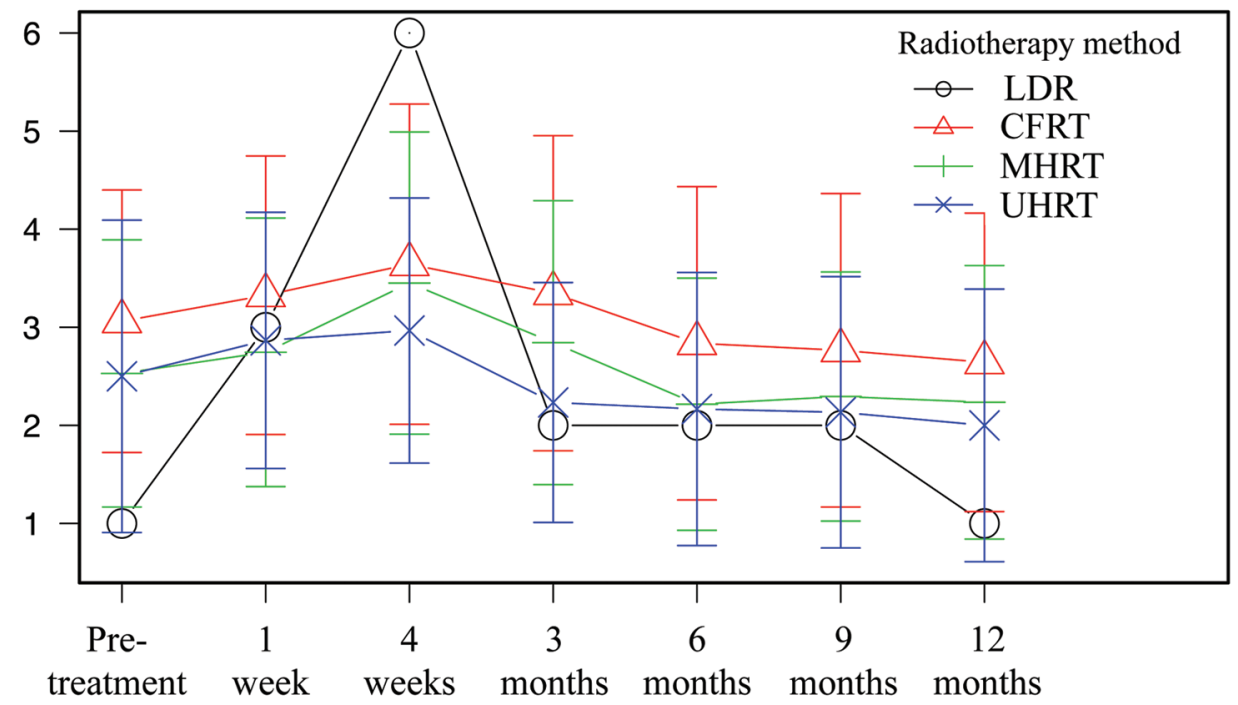

Figure 2. Changes in the QOL score according to radiotherapy methods. QOL: Quality of life; LDR: low-dose rate; CFRT: conventional fractionated radiotherapy; MHRT: moderate-hypofractionated radiotherapy; UHRT: ultra-hypofractionated radiotherapy.

and UHRT (20 cases, 40\%) groups. The details regarding acute toxicities are presented in Table II. Although the toxicities included duplication, urinary retention was the most common complaint among patients in the UHRT group, and its characteristics were similar to those of patients in the HDR-BT and LDR-BT groups rather than in those in the CFRT group.
IPSS and QOL score transition. IPSS and QOL score transitions are shown in Figures 1 and 2, respectively. Because the score data of patients in the HDR-BT group could not be obtained, they were excluded from the analysis, and comparisons were made among patients in the other four groups. Data of the patients in the LDR-BT group showed the 

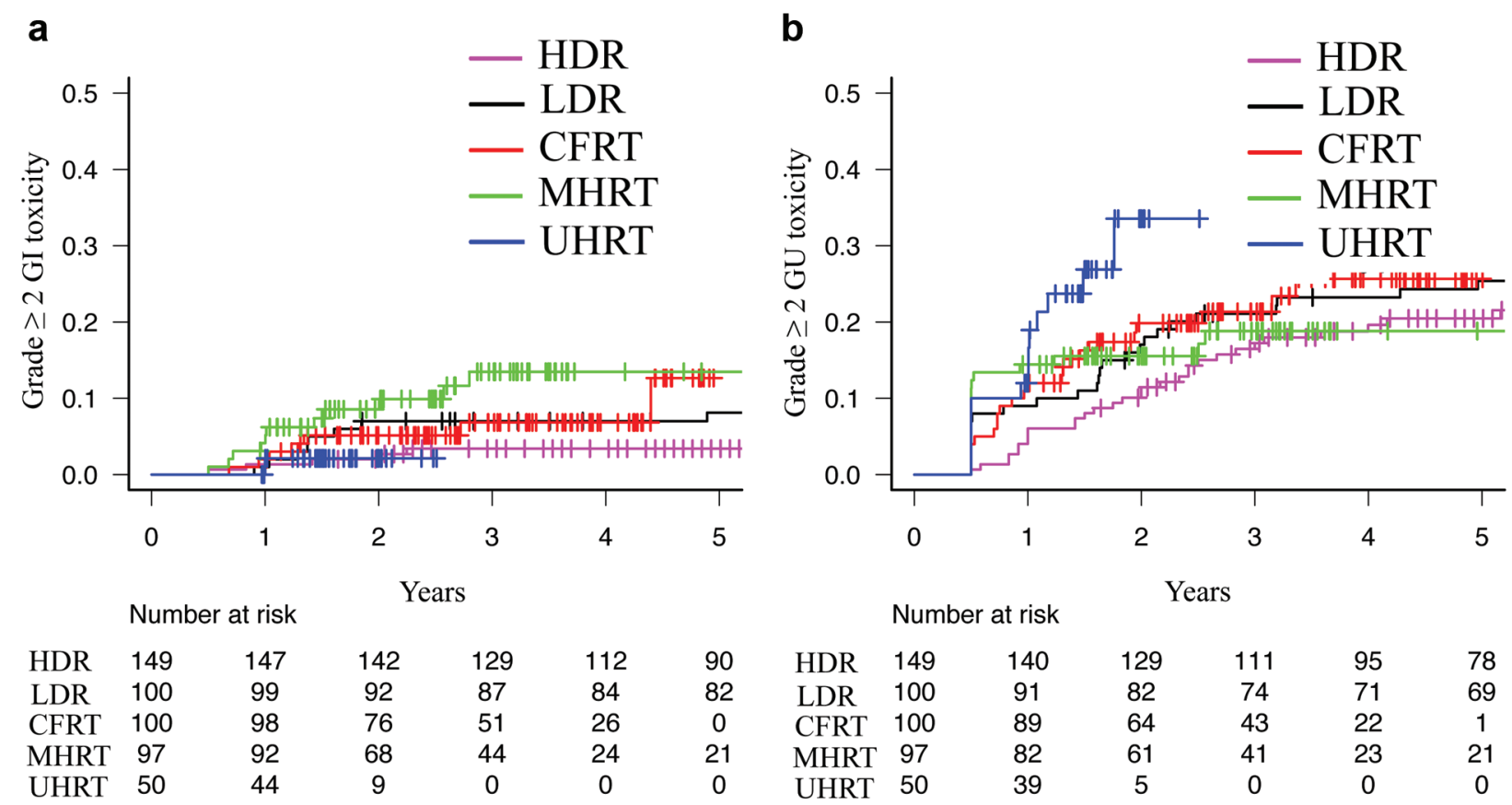

Figure 3. Cumulative incidence of grade $\geq 2 G I(A)$ and $G U(B)$ toxicities. GI: Gastrointestinal; GU: genitourinary; HDR: high-dose rate; LDR: low-dose rate; CFRT: conventional fractionated radiotherapy; MHRT: moderate-hypofractionated radiotherapy; UHRT: ultra-hypofractionated radiotherapy.

highest IPSS peak 4 weeks post-treatment, despite the pretreatment score being the lowest compared with that of patients in the other groups. A similar transition was observed for the QOL score. There was no difference in the degree of deterioration of the IPSS and QOL score after radiotherapy among the EBRT groups. However, the peaks of both scores occurred at the earliest for patients in the UHRT group, and accordingly, they peaked out at the earliest (at approximately 3 months). The scores in the three groups, in addition to the UHRT group, returned to baseline approximately 9 months after treatment.

Late toxicity. Because patients in the five radiotherapy groups had different follow-up periods, the cumulative incidence of late GI/GU toxicity was compared. Grade $\geq 2$ GI toxicity is shown in Figure $3 \mathrm{~A}$. The cumulative incidence of grade $\geq 2$ GI toxicity in patients in the HDR-BT group was marginally lower, but not significant $(p=0.053)$, than that in patients in the MHRT group. Only one patient had grade 3 GI toxicity: rectal haemorrhage in the LDR-BT group. The cumulative incidence of grade $\geq 2 \mathrm{GU}$ toxicity is shown in Figure 3B. The incidence of GU toxicity in patients in the UHRT group was high. There was a substantial difference in GU toxicity only in patients in the HDR-BT group $(p=0.005)$. Grade 3 haematuria developed in four patients: two in the HDR-BT group, one in the LDR-BT group, and one in the CFRT group. Furthermore, five cases of grade 3 urinary retention were observed in the HDR-BT group. No grade $\geq 4 \mathrm{GI} / \mathrm{GU}$ toxicities were observed.

The results of the univariate and multivariate analyses of late grade $\geq 2 \mathrm{GI} / \mathrm{GU}$ toxicity are shown in Table III. Regarding late grade $\geq 2$ GI, ADT administration tended to contribute but was not a significant factor. HDR-BT as a radiotherapy method was the only significant factor in the univariate analyses, and it remained significant in the multivariate analyses [hazard ratio $(\mathrm{HR})=0.36, p=0.04$ ]. In univariate and multivariate analyses, medication for bladder symptoms at radiotherapy $(\mathrm{HR}=1.91, p=0.009)$ and grade $\geq 2$ acute $\mathrm{GU}$ toxicity $(\mathrm{HR}=6.03, p<0.001)$ were significantly associated with late grade $\geq 2 \mathrm{GU}$ toxicity.

Grade $\geq 2$ erectile dysfunctions were observed in three $(2 \%)$, seven $(7 \%)$, one $(1 \%)$, four $(5 \%)$, and $0(0 \%)$ patients in the HDR-BT, LDR-BT, CFRT, MHRT, and UHRT groups, respectively. Two of the four patients in the MHRT group experienced grade 3 erectile dysfunction, without response to medication. Regarding another critical late toxicity, myelodysplastic syndromes (MDSs) were observed in one patient each in the CFRT and MHRT groups. Grade 2 MDS and grade 5 MDS were observed in the MHRT and CFRT groups, respectively. The patient in the CFRT group developed MDS 2.4 years after radiotherapy and died after 1.5 months. 
Table III. Univariate and multivariate analyses of late grade $\geq 2$ GI/GU toxicities.

\begin{tabular}{|c|c|c|c|c|c|c|}
\hline \multirow[t]{2}{*}{ Factor } & \multicolumn{3}{|c|}{ Univariate analysis } & \multicolumn{3}{|c|}{ Multivariate analysis } \\
\hline & HR & $95 \% \mathrm{CI}$ & $p$-Value & HR & $95 \% \mathrm{CI}$ & $p$-Value \\
\hline \multicolumn{7}{|l|}{ Late grade $\geq 2$ GI toxicity } \\
\hline Age & 1.01 & $0.96-1.07$ & 0.72 & & & \\
\hline iPSA & 0.96 & $0.91-1.01$ & 0.14 & & & \\
\hline Gleason score $(\geq 8)$ & 0.88 & $0.31-2.45$ & 0.81 & & & \\
\hline $\mathrm{T}$ classification (T1-T2 vs. T3-T4) & 0.54 & $0.17-1.78$ & 0.31 & & & \\
\hline ADT & 0.52 & $0.26-1.05$ & 0.07 & 0.57 & $0.28-1.18$ & 0.13 \\
\hline Duration before radiotherapy & 0.99 & $0.99-1.01$ & 0.38 & & & \\
\hline Total duration & 0.99 & $0.99-1.01$ & 0.43 & & & \\
\hline PTV volume & 1.01 & $0.98-1.02$ & 0.89 & & & \\
\hline Radiotherapy methods (HDR-BT vs. others) & 0.36 & $0.14-0.94$ & 0.04 & 0.36 & $0.14-0.94$ & 0.04 \\
\hline Acute grade $\geq 2$ GI & 2.15 & $0.51-8.98$ & 0.30 & & & \\
\hline \multicolumn{7}{|l|}{ Late grade $\geq 2$ GU toxicity } \\
\hline Age & 1.04 & $1.01-1.07$ & 0.02 & 1.02 & $0.98-1.07$ & 0.30 \\
\hline iPSA & 0.99 & $0.98-1.01$ & 0.47 & & & \\
\hline Gleason score $(\geq 8)$ & 1.57 & $0.41-2.40$ & 0.04 & 1.2 & $0.95-1.51$ & 0.13 \\
\hline $\mathrm{T}$ classification (T1-T2 vs. T3-T4) & 1.02 & $0.60-1.72$ & 0.94 & & & \\
\hline ADT & 1.13 & $0.74-1.70$ & 0.58 & & & \\
\hline Duration before radiotherapy & 0.99 & $0.99-1.01$ & 0.77 & & & \\
\hline Total duration & 1.00 & $0.99-1.01$ & 0.83 & & & \\
\hline PTV volume & 1.01 & $0.99-1.01$ & 0.21 & & & \\
\hline Radiotherapy methods (UHRT vs. others) & 2.18 & $1.21-3.99$ & 0.01 & 1.75 & $0.94-3.26$ & 0.07 \\
\hline Medication for bladder symptoms at radiotherapy & 2.64 & $1.65-4.22$ & $<0.001$ & 1.91 & $1.18-3.11$ & 0.009 \\
\hline Acute grade $\geq 2 \mathrm{GU}$ & 3.96 & $2.76-5.70$ & $<0.001$ & 6.03 & $3.56-10.2$ & $<0.001$ \\
\hline
\end{tabular}

HR: Hazard ratio; CI: confidence interval; GI: gastrointestinal; GU: genitourinary; HDR-BT: high-dose rate brachytherapy; UHRT: ultrahypofractionated radiotherapy; iPSA: initial prostate-specific antigen; ADT: androgen deprivation therapy; PTV: planning target volume; NA: not available.

\section{Discussion}

In this retrospective study of 496 prostate cancer patients from four institutions, no significant differences were observed in the treatment efficacies among the five radiotherapy methods. Regarding acute GU toxicity and late GI toxicity, patients in the HDR-BT group had better outcomes than those in the other groups. The IPSS and QOL scores based on patient reports showed the worst peaks in the LDR-BT group 4 weeks after radiotherapy. The IPSS peak occurred the earliest in the UHRT group, and the cumulative incidence of late GU toxicity was the highest despite the short follow-up period.

To the best of our knowledge, no study has directly compared the five radiotherapy methods for prostate cancer. While a few retrospective studies had already compared HDR-BT with LDR-BT, they showed a slight advantage of HDR-BT, especially regarding acute GU toxicity $(5,7,13)$. These study findings revealed that the HDR-BT group experienced less acute GU toxicity and late GI toxicity than those in the hypofractionated EBRT groups. Yamazaki et al. found that patients in the HDR-BT group had a lower incidence of grade $\geq 2$ acute GU toxicity with an equivalent incidence of late grade $\geq 2 \mathrm{GI} / \mathrm{GU}$ toxicities than patients in the LDR-BT group (5). Although the comparison between HDR-BT as monotherapy and CFRT is insufficient, a retrospective study indicated that grade 1 or 2 GI toxicity was not observed in the HDR-BT group (12). The frequency of grade 1 or 2 GI toxicity was significantly lower in the HDR-BT group than in the CFRT group. The results of our studies in the HDR-BT group are comparable with those of these previously published studies. We consider that the low toxicity of the HDR-BT technique is supported by low rectal and urethral doses (16).

A few reports have compared LDR-BT with CFRT, and they have commonly indicated that acute GU toxicities were more severe in LDR-BT than in CFRT $(8,13)$. In contrast, in our study, IPSS and QOL scores were worse in the LDR-BT group, while no significant difference was observed in physician-recorded acute GU toxicities between the LDR-BT and EBRT groups. We hypothesized that these seemingly paradoxical results were due to the use of prophylactic alphablocker medications. Only the LDR-BT group received prophylactic medication for approximately 1 year after 
radiotherapy, which probably resulted in underestimation of the acute GU toxicities. We believe that the patient-reported IPSS and QOL scores were accurate results, and that LDR-BT had a disadvantage over EBRT regarding acute GU toxicity.

Hypofractionated EBRT has gained increased attention owing to its proposed high radiation-fraction sensitivity or cost-effectiveness. After the CHHiP trial, MHRT was no longer the standard clinical treatment (10). Conversely, the clinical application of UHRT remained controversial, especially in terms of GU toxicity. The results of the HYPORT-PC study indicated that acute GU toxicities were more pronounced with UHRT than with CFRT (11). In contrast, the PACE-B trial reported that UHRT did not increase the incidence of acute toxicity compared with CFRT (2). In our study, the UHRT group was comparable to other EBRT groups regarding both physician-recorded toxicities and patient-reported outcomes in the acute phase. However, many GU toxicities were observed in approximately one year. The cumulative incidence of late GU toxicity was slightly higher in the UHRT group than in the other groups. Further accumulation of data and longer follow-up are warranted for the UHRT group to determine whether many events will occur at a later phase.

A comparison of toxicities, in addition to GI/GU, is also essential. In this study, two cases developed MDS after radiotherapy in the EBRT group, and one case of MDS was grade 5. Therapy-related MDS, defined as MDS occurring after previous chemotherapy or radiotherapy exposure, has accounted for $10 \%-20 \%$ of all MDS diagnoses (17). A retrospective cohort study of prostate cancer patients indicated that patients who underwent radiotherapy had a significantly increased risk of MDS (18). Importantly, further analysis of modalities confirmed an increased risk of developing MDS only with EBRT and not brachytherapy. Because prostate cancer has a good prognosis, a low incidence of secondary cancer is a unique advantage of brachytherapy.

This study has some limitations owing to its nonrandomised retrospective nature and multi-institutional analysis. Although evaluation criteria of physician-recorded toxicities were discussed in advance, there may have been bias among observers. The collected data had missing values. In particular, IPSS and QOL scores could not be obtained from patients in the HDR-BT group, and comparisons with patients in the other groups could not be performed. Although a linear accelerator and CyberKnife were used for EBRT, the physical characteristics differed (19). The difference could have been a confounding factor in the comparison of each fractionation in EBRT. Each treatment group had a different number of patients and a different follow-up period, especially the UHRT which had a small number and a brief follow-up period. A longer follow-up with higher numbers of patients is required.
Regardless of the risk stratification, no significant differences were observed in the biochemical tumour control rates among the five radiotherapy methods for prostate cancer. However, we observed a slight advantage of HDRBT over the other radiotherapy methods in terms of toxicity. Based on patient-reported outcomes, the toxicities were more severe in the LDR-BT group than in the EBRT group. The cumulative incidence of late GU toxicity was possibly the highest in the UHRT group. While the findings of this study can help determine the best radiotherapy method for the patient, further studies with more data and a longer followup period, are required.

\section{Conflicts of Interest}

The Authors have no conflicts of interest to declare in relation to this study.

\section{Authors' Contributions}

All Authors contributed to the study conception and design. Material preparation and data collection were performed by Makoto Ito, Kazuma Sasamura, Yuuki Takase, Tadayuki Kotsuma, Yukihiko Oshima, Yoshitaka Minami, Junji Suzuki, Eiichi Tanaka, Masahiko Oguchi, Takahito Okuda, Kojiro Suzuki, and Yasuo Yoshioka. All statistical analyses were performed by Makoto Ito and Wataru Ohashi. The first draft of the manuscript was written by Makoto Ito and all Authors commented on previous versions of the manuscript. All Authors read and approved the final manuscript.

\section{Acknowledgements}

The Authors would like to thank Editage (www.editage.com) for English language editing. This work was partially supported by Taiju Life Social Welfare Foundation and JSPS KAKENHI (grant number JP20K16741).

\section{References}

1 Zhou CK, Check DP, Lortet-Tieulent J, Laversanne M, Jemal A, Ferlay J, Bray F, Cook MB and Devesa SS: Prostate cancer incidence in 43 populations worldwide: An analysis of time trends overall and by age group. Int J Cancer 138(6): 1388-1400, 2016. PMID: 26488767. DOI: 10.1002/ijc.29894

2 Brand DH, Tree AC, Ostler P, van der Voet H, Loblaw A, Chu W, Ford D, Tolan S, Jain S, Martin A, Staffurth J, Camilleri P, Kancherla K, Frew J, Chan A, Dayes IS, Henderson D, Brown S, Cruickshank C, Burnett S, Duffton A, Griffin C, Hinder V, Morrison K, Naismith O, Hall E, van As N and PACE Trial Investigators: Intensity-modulated fractionated radiotherapy versus stereotactic body radiotherapy for prostate cancer (PACE-B): acute toxicity findings from an international, randomised, open-label, phase 3, non-inferiority trial. Lancet Oncol 20(11): 1531-1543, 2019. PMID: 31540791 . DOI: 10.1016/S1470-2045(19)30569-8

3 Mohler JL, Antonarakis ES, Armstrong AJ, D'Amico AV, Davis BJ, Dorff T, Eastham JA, Enke CA, Farrington TA, Higano CS, Horwitz EM, Hurwitz M, Ippolito JE, Kane CJ, Kuettel MR, 
Lang JM, McKenney J, Netto G, Penson DF, Plimack ER, PowSang JM, Pugh TJ, Richey S, Roach M, Rosenfeld S, Schaeffer E, Shabsigh A, Small EJ, Spratt DE, Srinivas S, Tward J, Shead DA and Freedman-Cass DA: Prostate cancer, version 2.2019, NCCN clinical practice guidelines in oncology. J Natl Compr Canc Netw 17(5): 479-505, 2019. PMID: 31085757. DOI: 10.6004/jncen.2019.0023

4 Goldner G, Pötter R, Battermann JJ, Kirisits C, Schmid MP, Sljivic S and van Vulpen M: Comparison between external beam radiotherapy (70 Gy/74 Gy) and permanent interstitial brachytherapy in 890 intermediate risk prostate cancer patients. Radiother Oncol 103(2): 223-227, 2012. PMID: 22398311. DOI: 10.1016/j.radonc.2012.01.016

5 Yamazaki H, Masui K, Suzuki G, Nakamura S, Yamada K, Okihara K, Shiraishi T, Yoshida K, Kotsuma T, Tanaka E, Otani $\mathrm{K}$, Yoshioka Y and Ogawa K: High-dose-rate brachytherapy monotherapy versus low-dose-rate brachytherapy with or without external beam radiotherapy for clinically localized prostate cancer. Radiother Oncol 132: 162-170, 2019. PMID: 30416045. DOI: 10.1016/j.radonc.2018.10.020

6 Yoshioka Y, Kotsuma T, Komiya A, Kariya S, Konishi K, Nonomura N, Ogawa K, Tanaka E, Nishimura K, Fujiuchi Y, Kitamura H, Yamagami T, Yamasaki I, Nishimura K, Teshima T, Nakamura K and Itami J: Nationwide, multicenter, retrospective study on high-dose-rate brachytherapy as monotherapy for prostate cancer. Int J Radiat Oncol Biol Phys 97(5): 952-961, 2017. PMID: 28333018. DOI: 10.1016/j.ijrobp.2016.12.013

7 Morgan TM, Press RH, Cutrell PK, Zhang C, Chen Z, Rahnema S, Sanda M, Pattaras J, Patel PR, Jani AB and Rossi PJ: Brachytherapy for localized prostate cancer in the modern era: a comparison of patient-reported quality of life outcomes among different techniques. J Contemp Brachytherapy 10(6): 495-502, 2018. PMID: 30662471. DOI: 10.5114/jcb.2018.81024

8 Tsubokura T, Yamazaki H, Masui K, Sasaki N, Shimizu D, Suzuki G, Nakamura S, Yamada K, Okihara K, Shiraishi T, Yoshida K, Nishikawa T and Okabe H: Comparison of imageguided intensity-modulated radiotherapy and low-dose rate brachytherapy with or without external beam radiotherapy in patients with localized prostate cancer. Sci Rep 8(1): 10538, 2018. PMID: 30002393. DOI: 10.1038/s41598-018-28730-1

9 Goy BW, Soper MS, Chang T, Slezak JM, Cosmatos HA and Tome M: Treatment results of brachytherapy vs. external beam radiation therapy for intermediate-risk prostate cancer with 10 year followup. Brachytherapy 15(6): 687-694, 2016. PMID: 27600607. DOI: $10.1016 /$ j.brachy.2016.06.015

10 Dearnaley D, Syndikus I, Mossop H, Khoo V, Birtle A, Bloomfield D, Graham J, Kirkbride P, Logue J, Malik Z, Money-Kyrle J, O'Sullivan JM, Panades M, Parker C, Patterson H, Scrase C, Staffurth J, Stockdale A, Tremlett J, Bidmead M, Mayles H, Naismith O, South C, Gao A, Cruickshank C, Hassan S, Pugh J, Griffin C, Hall E and CHHiP Investigators: Conventional versus hypofractionated high-dose intensitymodulated radiotherapy for prostate cancer: 5-year outcomes of the randomised, non-inferiority, phase $3 \mathrm{CHHiP}$ trial. Lancet Oncol 17(8): 1047-1060, 2016. PMID: 27339115. DOI: 10.1016/S1470-2045(16)30102-4

11 Widmark A, Gunnlaugsson A, Beckman L, Thellenberg-Karlsson C, Hoyer M, Lagerlund M, Kindblom J, Ginman C, Johansson B, Björnlinger K, Seke M, Agrup M, Fransson P, Tavelin B,
Norman D, Zackrisson B, Anderson H, Kjellén E, Franzén L and Nilsson P: Ultra-hypofractionated versus conventionally fractionated radiotherapy for prostate cancer: 5-year outcomes of the HYPO-RT-PC randomised, non-inferiority, phase 3 trial. Lancet 394(10196): 385-395, 2019. PMID: 31227373. DOI: 10.1016/S0140-6736(19)31131-6

12 Morimoto M, Yoshioka Y, Konishi K, Isohashi F, Takahashi Y, Ogata T, Koizumi M, Teshima T, Bijl HP, van der Schaaf A, Langendijk JA and Ogawa K: Comparison of acute and subacute genitourinary and gastrointestinal adverse events of radiotherapy for prostate cancer using intensity-modulated radiation therapy, three-dimensional conformal radiation therapy, permanent implant brachytherapy and high-dose-rate brachytherapy. Tumori 100(3): 265-271, 2014. PMID: 25076236. DOI: 10.1700/1578.17198

13 Strom TJ, Cruz AA, Figura NB, Shrinath K, Nethers K, Mellon EA, Fernandez DC, Saini AS, Hunt DC, Heysek RV and Wilder RB: Health-related quality-of-life changes due to high-dose-rate brachytherapy, low-dose-rate brachytherapy, or intensity-modulated radiation therapy for prostate cancer. Brachytherapy 14(6): 818825, 2015. PMID: 26452602. DOI: 10.1016/j.brachy.2015.08.012

14 Roach M 3rd, Hanks G, Thames H Jr, Schellhammer P, Shipley WU, Sokol GH and Sandler H: Defining biochemical failure following radiotherapy with or without hormonal therapy in men with clinically localized prostate cancer: recommendations of the RTOG-ASTRO Phoenix Consensus Conference. Int J Radiat Oncol Biol Phys 65(4): 965-974, 2006. PMID: 16798415. DOI: 10.1016/j.ijrobp.2006.04.029

15 Kanda Y: Investigation of the freely available easy-to-use software 'EZR' for medical statistics. Bone Marrow Transplant 48(3): 452458, 2013. PMID: 23208313. DOI: 10.1038/bmt.2012.244

16 Georg D, Hopfgartner J, Gòra J, Kuess P, Kragl G, Berger D, Hegazy N, Goldner G and Georg P: Dosimetric considerations to determine the optimal technique for localized prostate cancer among external photon, proton, or carbon-ion therapy and highdose-rate or low-dose-rate brachytherapy. Int J Radiat Oncol Biol Phys 88(3): 715-722, 2014. PMID: 24521685. DOI: 10.1016/j.ijrobp.2013.11.241

17 Borate U, Norris BA, Statler A, Fu R, Bucy T and Sekeres MA: Representation of therapy-related myelodysplastic syndrome in clinical trials over the past 20 years. Blood Adv 3(18): 2738-2747, 2019. PMID: 31537526 . DOI: 10.1182/bloodadvances. 2019000293

18 Wang R, Zeidan AM, Yu JB, Soulos PR, Davidoff AJ, Gore SD, Huntington SF, Gross CP and Ma X: Myelodysplastic syndromes and acute myeloid leukemia after radiotherapy for prostate cancer: A population-based study. Prostate 77(5): 437-445, 2017. PMID: 27868212. DOI: 10.1002/pros.23281

19 Ito M, Kawamura T, Mori Y, Mori T, Takeuchi A, Oshima Y, Nakamura K, Aoyama $\mathrm{T}$, Kaneda $\mathrm{N}$, Ishiguchi $\mathrm{T}$ and Mizumatsu S: Dose distributions of high-precision radiotherapy treatment: A comparison between the cyberknife and truebeam systems. Int J Radiat Res 16(4): 395-402, 2018. DOI: 10.18869/acadpub.ijrr.16.4.395
Received March 18, 2021

Revised April 11, 2021

Accepted April 12, 2021 